\title{
Generation of $10^{15}-10^{17} \mathrm{eV}$ photons by UHE $\mathrm{CR}$ in the Galactic magnetic filed.
}

\author{
S. L. Dubovsky円, P. G. Tinyakov² \\ Institute for Nuclear Research of the Russian Academy of Sciences, 117312 \\ Moscow, Russia
}

\begin{abstract}
We show that the deep expected in the diffuse photon spectrum above the threshold of $e^{+} e^{-}$-pair production, i.e., at energies $10^{15}-$ $10^{17} \mathrm{eV}$, may be absent due to the synchrotron radiation by the electron component of the extragalactic Ultra-High Energy Cosmic Rays (UHE CR) in the Galactic magnetic filed. The mechanism we propose requires small $\left(<2 \times 10^{-12} \mathrm{G}\right)$ extragalactic magnetic fields and large fraction of photons in the UHE CR. For a typical photon flux expected in top-down scenarios of UHE CR, the predicted flux in the region of the deep is close to the existing experimental limit. The sensitivity of our mechanism to the extragalactic magnetic field may be used to improve existing bounds on the latter by two orders of magnitude.
\end{abstract}

\section{Introduction}

The spectrum of diffuse photons is expected to have a deep of more than two orders of magnitude at energies $10^{15}-10^{17} \mathrm{eV}$ [1]. This deep is similar in nature to the well-known Greisen-Zatsepin-Kuzmin (GZK) cutoff [2] and is caused by electron pair production on the cosmic microwave background, $\gamma \gamma_{b} \rightarrow e^{+} e^{-}$. The cross section of the latter process reaches its maximum of $0.3 \sigma_{T} \sim 0.2$ barn near the threshold at $3 \times 10^{14} \mathrm{eV}$ and decreases at higher energies (see, e.g., ref. [3]). The attenuation length of photons in the region of the deep is of order $100 \mathrm{kpc}$, so the deep in the spectrum is a universal feature of models in which high energy photons have extragalactic origin. Indeed, the existence of the deep is confirmed by simulations in various models of UHE CR (for a review see, e.g., refs. [3, 4). For instance,

\footnotetext{
${ }^{1}$ E-mail: sergd@ms2.inr.ac.ru

${ }^{2}$ E-mail: peter@ms2.inr.ac.ru
} 
in the region of the deep the top-down models typically give the photon flux of order $10^{-3} \mathrm{eV} \mathrm{cm}^{-2} \mathrm{~s}^{-1} \mathrm{sr}^{-1}$, while at ultra-high energies, $E \gtrsim 10^{20} \mathrm{eV}$, the predicted flux is more than two orders of magnitude larger and reaches (a few) $\times 10^{-1} \mathrm{eV} \mathrm{cm}^{-2} \mathrm{~s}^{-1} \mathrm{sr}^{-1}$.

The spectrum of diffuse photons at energies above $\sim 10^{11} \mathrm{eV}$ is known rather poorly. In the region of the deep the bounds have been obtained by EAS-TOP [5] and CASA-MIA [6]. At $E \sim 10^{16} \mathrm{eV}$ the bound is of order $\sim 0.5 \mathrm{eV} \mathrm{cm}^{-2} \mathrm{~s}^{-1} \mathrm{sr}^{-1}$, about two orders of magnitude higher than has been predicted by top-down models. At ultra-high energies the photon flux can be as large as $\sim 1 \mathrm{eV} \mathrm{cm}^{-2} \mathrm{~S}^{-1} \mathrm{sr}^{-1}$ if the observed UHE CR events are interpreted as photons (recent results from AGASA [7] suggest this possibility [8]).

While experimentally the existence of the deep in the photon spectrum is an open question, theoretically it is not solid either. Calculations of the spectrum cited above do not take into account the possibility that high energy photons can be generated in our Galaxy by UHE electrons via synchrotron radiation in the Galactic magnetic field. As we argue below, the account for synchrotron emission may substantially change the photon spectrum at $E \sim 10^{15}-10^{17} \mathrm{eV}$ filling the deep and bringing the expected photon flux close to the existing experimental limit.

The synchrotron mechanism requires large flux of UHE electrons to hit the Galactic magnetic field. It has been recently pointed out in ref. 90 that this condition is naturally satisfied in the halo models of UHE CR (these models explain UHE CR by decays of heavy relic particles clustered in the Galactic halo [10]). Due to the fragmentation process, the decay products of the superheavy particles contain a large fraction of UHE electrons.

In this paper we show that UHE electrons which are necessary for the synchrotron mechanism to work can be of extragalactic origin, provided extragalactic magnetic fields are small. We will see that, in fact, the large flux of UHE electrons is inherent in top-down models of UHE CR, so that the generation of high energy photons by the synchrotron mechanism is a generic prediction of top-down scenarios and is not specific to halo models of UHE CR .

The key observation is that UHE photons propagate in the extragalactic space via cascade process being converted to electrons and back with a small

\footnotetext{
${ }^{3}$ Throughout this paper the "flux" denotes the quantity which is expressed in terms of th edifferential spectrum $j(E)$ by means of the relation $F=E^{2} j(E)$.
} 
energy loss. As a result, the flux of UHE photons is necessarily accompanied by the flux of UHE electrons. At energies of order $10^{22}-10^{23} \mathrm{eV}$ and in the absence of extragalactic magnetic fields the electron flux is at least as large, or even much larger, than the photon onef. While UHE photons reach the Earth and contribute to the observable flux of UHE CR, UHE electrons emit synchrotron radiation in the Galactic magnetic field and transfer their energy to high energy photons. As we will see below, the energy of produced photons lies in the region of the deep, while their flux is similar to that of UHE electrons (and, thus, of UHE photons). Hence, in the absence of extragalactic magnetic fields the flux of UHE photons at the level of $\sim 1 \mathrm{eV} \mathrm{cm}^{-2} \mathrm{~s}^{-1} \mathrm{sr}^{-1}$ implies the flux of synchrotron photons at the same level which fills the deep in the photon spectrum. Since the large flux of UHE photons is one of the signatures of the top-down mechanisms of UHE CR, in the absence of extragalactic magnetic fields these models generically predict no deep in the spectrum of diffuse photons.

The mechanism we propose is sensitive to magnetic fields on distances up to $50 \mathrm{Mpc}$ from our Galaxy. If no deep in the photon spectrum is observed and halo models are ruled out, the extragalactic magnetic field on this distances must be smaller than $2 \times 10^{-12} \mathrm{G}$. This is two orders of magnitude better than the existing bounds [11]. Inversely, if the deep is found and, at the same time, UHE CR have a large fraction of photons, the extragalactic magnetic field must be larger than $2 \times 10^{-12} \mathrm{G}$.

The paper is organized as follows. In Sect. 2 we estimate the flux of UHE electrons given the flux of UHE photons and zero extragalactic magnetic field. In Sect. 3 we calculate the spectrum of synchrotron radiation in the galactic magnetic field for injected electrons of given energy. In Sect.4 we estimate the effect of extragalactic magnetic fields. Sect.5 contains our conclusions.

\section{The flux of UHE electrons.}

Our aim in this section is to show that in the energy range $10^{22}-10^{23} \mathrm{eV}$ the flux of UHE photons is necessarily accompanied by a comparable or larger flux of UHE electrons, provided the extragalactic magnetic fields are absent. The argument is based on the observation that at these energies the photon

\footnotetext{
${ }^{4}$ In the presence of extragalactic magnetic field the electrons rapidly loose energy via synchrotron radiation and the argument may not work (see Sect.4 for details).
} 
propagation is a cascade process (see, e.g., [1]), i.e., propagating photon is converted to an electron and back with small energy loss. As this process is random, one should expect certain ratio of photons and electrons far from the source.

The main reactions driving the cascade are $e^{+} e^{-}$pair production $(\mathrm{PP})$ on the radio background, $\gamma \gamma_{b} \rightarrow e^{+} e^{-}$, double pair production (DPP), $\gamma \gamma_{b} \rightarrow$ $e^{+} e^{-} e^{+} e^{-}$, and the inverse Compton scattering (ICS), $e \gamma_{b} \rightarrow e \gamma$ [3]. In the energy range of interest double pair production dominates, so one should expect to find more electrons than photons.

A simple estimate can be obtained if one neglects secondary particles and energy losses. In this (rather crude) approximation PP and DPP lead to the conversion of photon to electron with the rates $a_{P P}$ and $a_{D P P}$, respectively, while ICS converts electron back to photon with the rate $b$. The set of equations which describes propagation of photons and electrons reads

$$
\begin{aligned}
& \frac{d n_{\gamma}}{d R}=-a n_{\gamma}+b n_{e}, \\
& \frac{d n_{e}}{d R}=a n_{\gamma}-b n_{e},
\end{aligned}
$$

where $R$ is the distance from the source, $n_{\gamma}(R)$ and $n_{e}(R)$ are fractions of photons and electrons at the distance $R$, respectively, and $a \equiv a_{P P}+a_{D P P}$. The solution to this system is

$$
\frac{n_{e}}{n_{\gamma}}=\frac{a \mathrm{e}^{R(a+b)}-C}{b \mathrm{e}^{R(a+b)}+C}
$$

where $C$ is an integration constant whose value is determined by the ratio $n_{e} / n_{\gamma}$ at $R=0$. Far from the source the value of this constant is irrelevant.

The observed fluxes $F_{e, \gamma}$ are given by integrals over the space of $n_{e, \gamma}$ multiplied by the particle injection rate. To estimate $F_{e} / F_{\gamma}$ we note that the integrals are dominated by large distances where the ratio $n_{e} / n_{\gamma}$ is constant,

$$
\frac{n_{e}}{n_{\gamma}} \sim \frac{a}{b} .
$$

Therefore,

$$
\frac{F_{e}}{F_{\gamma}} \sim \frac{a}{b} .
$$


Both $a$ and $b$ depend on energy. At $E \sim 10^{22} \mathrm{eV}$ one has $a \sim 2 \times 10^{-2}$ $\mathrm{Mpc}^{-1}, b \sim 8 \times 10^{-3} \mathrm{Mpc}^{-1}$ [3], and thus

$$
\frac{F_{e}}{F_{\gamma}} \sim 2 \quad \text { at } \quad E=10^{22} \mathrm{eV}
$$

At higher energies the rate $a$ is dominated by DPP process and tends to a constant, $a \rightarrow 8 \times 10^{-3} \mathrm{Mpc}^{-1}$, while the rate $b$ rapidly falls off [3]. At $E \sim 10^{23} \mathrm{eV}$ eq.(2) gives

$$
\frac{F_{e}}{F_{\gamma}} \sim 10 \quad \text { at } \quad E=10^{23} \mathrm{eV}
$$

In a more accurate estimate one should take into account energy losses by leading particles and possible energy sharing in the leading $e^{+} e^{-}$-pair in DPP. In this approximation the result depends on the energy distribution of initial particles, as well as on the energy dependence of the rates $a_{P P}, a_{D P P}$ and $b$. We have performed such estimate by dividing energy interval $10^{21}-10^{24}$ $\mathrm{eV}$ into 10 energy bands and solving numerically the system of 20 coupled equations analogous to eqs.(1). We have found that the corrections to eqs.(3) and (4) are small and do not change our conclusions, unless the extragalactic magnetic filed is non-zero (the latter case is considered in Sect.4).

\section{Synchrotron radiation in the galactic mag- netic field.}

Consider now the synchrotron radiation of UHE electrons in the Galactic magnetic field. An ultra-relativistic particle of energy $E$ moving in the magnetic field $B$ emits radiation at the characteristic frequency 12

$$
\omega_{c}=\frac{3 \sqrt{\alpha} B}{2 m_{e}^{3}} E^{2}=6.7 \times 10^{14}\left(\frac{E}{10^{20} \mathrm{eV}}\right)^{2}\left(\frac{B}{10^{-6} \mathrm{G}}\right) \mathrm{eV} .
$$

The width of the frequency band is roughly $\delta \omega \sim \omega_{c}$. As a result of this process, the particle looses energy at the rate

$$
\frac{d E}{d x}=-\frac{2 \alpha^{2} B^{2}}{3 m^{4}} E^{2}
$$


Both equations are written for the case of particle momentum normal to the direction of the magnetic field. Generalization to other cases is straightforward.

Eq.(5) implies that in the Galactic magnetic field, $B \sim 10^{-6} \mathrm{G}$, electrons with energy $E \sim 10^{20} \mathrm{eV}$ radiate at the characteristic frequency $\omega_{c} \sim 10^{15} \mathrm{eV}$. This process is the source of high energy photons in the Galactic halo models of UHE CR [9]. Since the Galactic magnetic field far from the Galactic center is smaller, the extragalactic electrons which we consider in this paper should have higher energy in order to produce synchrotron radiation in the same frequency range.

For the quantitative analysis of the synchrotron emission by the extragalactic electrons consider an UHE electron moving in a varying magnetic field $B(x)$ perpendicular to its velocity. Integration of eq.(6) gives

$$
\frac{1}{E(x)}-\frac{1}{E_{0}}=\frac{2 \alpha^{2}}{3 m_{e}^{4}} \int_{-\infty}^{x} B^{2}(x) d x,
$$

where $E_{0}$ is the initial energy of the electron.

For definiteness, let us take the exponentially decaying magnetic field,

$$
B(x)=B_{0} \exp \left(x / x_{0}\right)
$$

(note that we consider a particle propagating from $x=-\infty$ ). This behavior is expected in some recent Galactic magnetic field models [13] for the field in the direction normal to the Galactic disk. The scale $x_{0}$ is of order $4 \mathrm{kpc}$. Making use of eqs.(7) and (8) one finds the relation between $E$ and $B$ at a given point of particle trajectory,

$$
\frac{1}{E}-\frac{1}{E_{0}}=\frac{\alpha^{2} x_{0}}{3 m_{e}^{4}} B^{2} .
$$

This equation, together with eq.(5), determines the dominant radiation frequency as a function of particle energy,

$$
\omega_{c}(E)=\frac{9 E_{0}^{3 / 2}}{2 m_{e} \sqrt{3 \alpha x_{0}}} f\left(E / E_{0}\right),
$$

where

$$
f(y)=y^{3 / 2} \sqrt{1-y}
$$




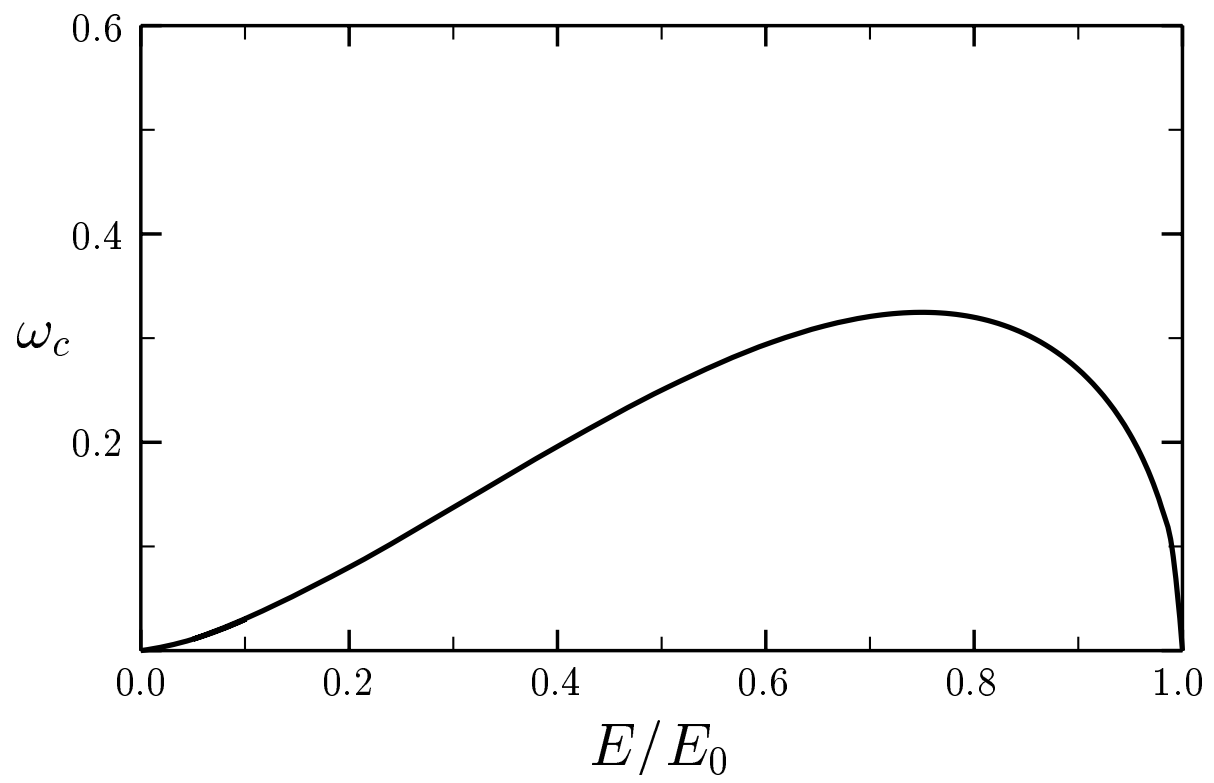

Figure 1: The dependence of $\omega_{c}$ (arbitrary units) on particle energy for the exponential model of the Galactic magnetic field.

This function is shown in Fig.1. As can be seen from the picture, most part of the electron energy is emitted at frequencies close to

$$
\omega_{\max }=\omega_{c}\left(3 E_{0} / 4\right)=\frac{27 E_{0}^{3 / 2}}{32 m_{e} \sqrt{\alpha x_{0}}}=0.8 \times 10^{15}\left(\frac{E_{0}}{10^{22} \mathrm{eV}}\right)^{3 / 2}\left(\frac{x_{0}}{4 \mathrm{kpc}}\right)^{-1 / 2} \mathrm{eV}
$$

According to eqs.(可) and (9), at $E=10^{22} \mathrm{eV}$ the electron looses most part of its energy in the region where the magnetic field is $\sim 10^{-10} \mathrm{G}$, i.e., at the distance $\sim 36 \mathrm{kpc}$ from the galactic disk for $B_{0} \sim 10^{-6} \mathrm{G}$.

In the case of magnetic field not perpendicular to particle velocity, the spectrum of synchrotron photons is softer. The same is true for the magnetic field which falls off slower than in eq.(8), as usually assumed for the Galactic magnetic filed in the direction parallel to the Galactic plane. Thus, one should expect angular dependence of photon spectrum with more energetic photons coming from the direction normal to the Galactic plane, and softer spectrum from the directions in the Galactic plane. 
Finally, let us estimate the flux of synchrotron photons assuming the flux of UHE photons which is typical for top-down scenarios, (a few) $\times$ $10^{-1} \mathrm{eV} \mathrm{cm}^{-2} \mathrm{~s}^{-1} \mathrm{sr}^{-1}$ at energies $\sim 10^{22}-10^{23} \mathrm{eV}$. Eq.(3) implies that, outside the Galactic magnetic field, there is at least as large flux of UHE electrons which transfer their energy to high energy photons in the Galactic magnetic field. Since the synchrotron spectrum has $\delta \omega \sim \omega$, the energy conservation implies that the flux of synchrotron photons is approximately the same as the flux of UHE electrons, which is larger by the factor $F_{e} / F_{\gamma}$ than the flux of UHE photons.

\section{Effect of extragalactic magnetic field}

As it was shown above, in the absence of extragalactic magnetic fields the observed flux of $10^{15}-10^{17} \mathrm{eV}$ photons is proportional to the flux of UHE photons and the ratio $F_{e} / F_{\gamma}$ at energies above $E \gtrsim 10^{22} \mathrm{eV}$. The presence of large enough extragalactic magnetic field can significantly decrease this ratio. Indeed, if $\gamma \rightarrow e$ conversion length, $a^{-1}=\left(a_{P P}+a_{D P P}\right)^{-1}$, is large compared to the energy loss length of the electron due to the synchrotron radiation in the extragalactic magnetic field, the flux of UHE electrons should be much smaller than the flux of UHE photons.

Let us estimate the value of the extragalactic magnetic field at which the ratio $F_{e} / F_{\gamma} \sim 1$ at $E \gtrsim 10^{22} \mathrm{eV}$. For this purpose note that the solution to eq.(6) in the constant magnetic field $B$ can be written in the form

$$
l=\frac{3 m^{4}}{2 \alpha^{2} B^{2} E}\left(1-\frac{E}{E_{0}}\right),
$$

where $l$ is the distance passed by the electron while its energy decreases from $E_{0}$ to $E$. Eq.(10) implies that electrons with energy $E$ can only come from distances smaller than

$$
l_{E}=\frac{3 m^{4}}{2 \alpha^{2} B^{2} E} \sim 50\left(\frac{2 \times 10^{-12} \mathrm{G}}{B}\right)^{2}\left(\frac{10^{22} \mathrm{eV}}{E}\right) \mathrm{Mpc} .
$$

Taking into account that the length $a^{-1}$ of $e \rightarrow \gamma$ conversion is of order $50 \mathrm{Mpc}$ at $E=10^{22} \mathrm{eV}$ [3] one finds that the extragalactic magnetic field should be smaller than $2 \times 10^{-12} \mathrm{G}$ at the distances $\leq 50 \mathrm{Mpc}$ from our 
Galaxy in order that the ratio $F_{e} / F_{\gamma}$ to be comparable or larger than one. If the magnetic field at distances of order $50 \mathrm{Mpc}$ is noticeably larger than $2 \times 10^{-12} \mathrm{G}$, the ratio $F_{e} / F_{\gamma}$ is much smaller than one and the flux of UHE electrons is not sufficient to fill the deep in photon spectrum by synchrotron mechanism (for the discussion of present limits on the extragalactic magnetic field see, e.g., ref. [14]).

It is worth noting that the mechanism we propose is not sensitive to magnetic fields at distances larger than $\sim 50 \mathrm{Mpc}$ because this distance is sufficient for generation of a large fraction of electrons, $F_{e} / F_{\gamma} \sim 1$. The effect of distant magnetic fields is mere decreasing of the UHE photon flux, which is not important for our argument since we normalize UHE photon flux to the observed flux of UHE CR.

\section{Conclusions}

To summarize, we have shown that the expected deep in the photon spectrum at energies $10^{15}-10^{17} \mathrm{eV}$ may be absent due to the synchrotron radiation of UHE CR in the Galactic magnetic field. The mechanism we propose involves extragalactic UHE electrons produced during cascade propagation of UHE photons and thus requires small extragalactic magnetic fields and large fraction of photons in UHE CR at energies $10^{22}-10^{23} \mathrm{eV}$. The latter is the characteristic feature of all top-down models of UHE CR. Note, however, that our mechanism does not rely on any peculiarities of these models.

When synchrotron radiation in the Galactic magnetic field is taken into account, the top-down models predict the flux of $10^{15}-10^{17} \mathrm{eV}$ photons which is close to the current experimental limits, provided that extragalactic magnetic fields are small. Thus, it is important to improve the sensitivity of the experiments in the energy range $10^{15}-10^{17} \mathrm{eV}$. The detection of the diffuse photon flux at the level of $\sim 10^{-1} \mathrm{eV} \mathrm{cm}^{-2} \mathrm{~s}^{-1} \mathrm{sr}^{-1}$ would strongly suggest that UHE CR are produced by a top-down mechanism. Moreover, this would imply that either the mechanism based on the halo model 99 or the one proposed here works. The two possibilities can be distinguished by measuring the angular anisotropy of UHE CR [15] and of produced high energy photons [9]. Additional signature of our mechanism is the dependence of the photon spectrum on the arrival direction as discussed in Sect.3. On the contrary, if the photon flux in the region of the deep is smaller than 
$\sim 10^{-3} \mathrm{eV} \mathrm{cm}^{-2} \mathrm{~s}^{-1} \mathrm{sr}^{-1}$ and, at the same time, UHE CR have a large fraction of photons, the extragalactic magnetic field must be larger than $2 \times 10^{-12} \mathrm{G}$.

\section{Acknowledgments}

The authors are indebted to D.S. Gorbunov, O.E. Kalashev, M.V. Libanov, V.A. Rubakov and D.V. Semikoz for useful discussions and comments. The work of S.D. is supported in part by Russian Foundation for Basic Research under grant 99-02-18410, INTAS grant 96-0457 within the research program

of the International Center for Fundamental Physics in Moscow, and ISSEP fellowship.

\section{References}

[1] R.J. Could, G. Schreder, Phys. Rev. Lett. 16 (1966) 252;

J.P. Jelley, Phys. Rev. Lett. 16 (1966) 479.

[2] K. Greisen, Phys. Rev. Lett. 16 (1966) 748;

G.T. Zatsepin and V.A. Kuzmin, Pisma Zh. Eksp. Teor. Fiz. 4 (1966) 144 ;

[3] S. Lee, Phys. Rev. D58 (1998) 043004;

[4] P. Bhattacharjee, G. Sigl, Origin and Propagation of Extremely High Energy Cosmic Rays, astro-ph/9811011;

[5] M. Aglietta et al., Astropart. Phys. 6 (1996) 71;

[6] M.C. Chantel et al., Phys. Rev. Lett.79 (1997) 1805;

[7] M. Takeda et al., Phys. Rev. Lett. 81 (1998) 1163, astro-ph/9807193.

[8] M. Teshima, talk presented at 10-th International School "Particles and Cosmology", Baksan, 1999.

[9] P. Blasi, Gamma Rays from Superheavy Relic Particles in the Halo, astro-ph/9901390; 
[10] V.A. Kuzmin and V.A. Rubakov, Phys. Atom. Nucl. 61 (1998) 1028; V. Berezinsky, M. Kachelriess and A. Vilenkin, Phys. Rev. Lett. 79 (1997) 4302;

[11] P.P. Kronberg, Rep. Prog. Phys., 47 (1994) 325.

[12] L.D. Landau and E.M. Lifshits, "Field Theory", Moscow, 1973.

[13] T. Stanev, Astrophys. J., 479 (1997) 290.

[14] D. Ryu, H. Kang, P.L. Biermann, A\&A, 335 (1998) 19, astroph/9803275;

T.A. Enßlin, P.L. Biermann, P.P. Kronberg, and X.P. Wu; Astrophysical Journal 477 (1997) 560, astro-ph/9609190;

[15] S.L. Dubovsky, P.G. Tinyakov, Pisma Zh. Eksp. Teor. Fiz., 68 (1998) 99. 